Algebraic 85 Geometric Topology

Volume 2 (2002) 449-463

Published: 30 May 2002

ATG

\title{
Framed holonomic knots
}

\author{
TOBIAS EKHOLM \\ MaXime WolfF
}

\begin{abstract}
A holonomic knot is a knot in 3-space which arises as the 2-jet extension of a smooth function on the circle. A holonomic knot associated to a generic function is naturally framed by the blackboard framing of the knot diagram associated to the 1 -jet extension of the function. There are two classical invariants of framed knot diagrams: the Whitney index (rotation number) $W$ and the self linking number $S$.

For a framed holonomic knot we show that $W$ is bounded above by the negative of the braid index of the knot, and that the sum of $W$ and $|S|$ is bounded by the negative of the Euler characteristic of any Seifert surface of the knot.

The invariant $S$ restricted to framed holonomic knots with $W=m$, is proved to split into $n$, where $n$ is the largest natural number with $n \leq \frac{|m|}{2}$, integer invariants. Using this, the framed holonomic isotopy classification of framed holonomic knots is shown to be more refined than the regular isotopy classification of their diagrams.
\end{abstract}

AMS Classification 57M27; 58C25

Keywords Framing, holonomic knot, Legendrian knot, self-linking number, Whitney index

\section{Introduction}

\section{A Holonomic knots and framing}

Let $f: S^{1} \rightarrow \mathbb{R}$ be a smooth function. The holonomic plane curve and holonomic space curve associated to $f$ are the 1-jet extension $c$ and the 2-jet extension $C$ of $f$, respectively. That is, $c(t)=\left(f(t), f^{\prime}(t)\right)$, and $C(t)=$ $\left(f(t), f^{\prime}(t), f^{\prime \prime}(t)\right)$ where $t \in S^{1}$, and where $\left(x_{0}, x_{1}\right)$ and $\left(x_{0}, x_{1}, x_{2}\right)$ are linear coordinates on $\mathbb{R}^{2}$ and $\mathbb{R}^{3}$, respectively.

A holonomic knot is a holonomic space curve which is an embedding. A framed holonomic knot is a holonomic knot with associated holonomic plane curve 
which is an immersion. If $f$ is a function giving rise to a framed holonomic knot $C$ then $\left(f^{\prime}(t), f^{\prime \prime}(t)\right) \neq 0$, for all $t \in S^{1}$, and we consider the constant vector field $\partial_{x_{2}}$ as a normal vector field along $C$.

In the space of smooth functions on the circle, the functions with associated holonomic space curve being a (framed) holonomic knot form an open and dense subset. A (framed) holonomic isotopy is a continuous 1-parameter family of (framed) holonomic knots, or equivalently a continuous path in the space of (framed) holonomic knots.

Vassiliev [5] introduced holonomic knots and proved that any knot class (topological isotopy class of knots) has a holonomic representative and also that there exists a natural isomorphism from finite type invariants of topological knots to finite type invariants of holonomic knots.

Birman and Wrinkle [2] showed that two holonomic knots which are topologically isotopic are in fact holonomically isotopic. From a combinatorial point of view this means that the holonomic isotopy classification of holonomic knots is identical to the isotopy classification of their diagrams. (A knot diagram is the image of a generic projection of a knot to a plane in $\mathbb{R}^{3}$, decorated with over and under crossing information at its double points. An isotopy of a knot digram is defined to be a sequence of planar isotopies and Reidemeister moves, see e.g. Kauffman [3].)

\section{B Whitney index and self linking number}

Following Kauffman [3], we say that two knot diagrams which can be deformed into each other by a sequence of planar isotopies and, second- and third Reidemeister moves (i.e. the moves the projections of which are self-tangency- and triple point instances) are called regularly isotopic. There are two simple invariants of regular isotopy:

Fix an orientation of the ambient $\mathbb{R}^{3}$. This orientation together with a fixed orientation of the projection direction associated to the knot diagram induce an orientation on the projection plane. The Whitney index $W$ is the tangential degree of the knot diagram viewed as an oriented regular plane curve in the projection plane. The self linking number $S$ is the linking number of a knot $K$ which projects to the diagram and a copy of $K$ shifted slightly in the projection direction, computed using the fixed orientation of the ambient $\mathbb{R}^{3}$.

The Whitney formula [6] expresses the Whitney index of a generic regular plane curve as follows. Let $q$ be a point on $C$ such that $C$ lies on one side of the 
tangent line of $C$ at $q$. Let $\mu(q)= \pm 1$ denote the winding number of $C$ with respect to $q^{\prime}$, where $q^{\prime}$ is the point $q$ shifted slightly into the half plane which contains the curve. The orientation of $C$ and the point $q$ induces an ordering of the preimages of a self intersection point $p$ of $C$. Let $\epsilon_{p}= \pm 1$ be the sign of the orientation of the plane induced from the tangent vectors of the ordered branches of $C$ intersecting at $p$. Then

$$
W(C)=-\sum_{p} \epsilon(p)+\mu(q) .
$$

The self linking number is the sum of the crossing signs over all crossings of the diagram. Hence $W+S$ is an odd integer.

In our study of framed holonomic knots we use the orientation $d x_{0} \wedge d x_{1} \wedge d x_{2}>$ 0 and project along the $x_{2}$-axis oriented by $d x_{2}>0$ to define $W$ and $S$ for framed holonomic knots. The ranges of these invariants are easily found:

Proposition 1.1 Let $C$ be a framed holonomic knot. Then $W(C)<0$, and if $W(C)=-1$ then $S(C)=0$ and $C$ represents the unknot. Let $m \leq-2$ and $n \in \mathbb{Z}$ be such that $m+n$ is odd. Then there exists a framed holonomic knot with $W(C)=m$ and $S(C)=n$.

Proposition 1.1 is proved in Subsection 2.A.

It is more interesting to consider the ranges of $W$ and $S$ restricted to diagrams representing a fixed knot class $K$. It is easy to see that for any integers $m$ and $n$ such that $m+n$ is odd there exists a diagram $D$ which represents $K$ with $W(D)=m$ and $S(D)=n$. If the domains of $W$ and $S$ are restricted further to framed holonomic knots which represent $K$ the situation changes drastically.

Theorem 1.2 Let $C$ be a framed holonomic knot representing the knot class $K$. Then

$$
W(C) \leq-\operatorname{braid}(K),
$$

where $\operatorname{braid}(K)$ is the braid index of $K$, and

$$
W(C)+|S(C)| \leq 2 g(K)-1,
$$

where $g(K)$ denotes the genus of $K$.

Theorem 1.2 is proved in Subsection 3.C. The proof of (1.2) uses the Bennequin inequality [1] from the theory of Legendrian knots: if $(x, y, z)$ are coordinates on $\mathbb{R}^{3}$ and $\mathbb{R}^{3}$ is oriented by $d x \wedge d y \wedge d z>0$ then this inequality asserts that 
for the $x y$-diagram $\Gamma_{\mathbb{C}}$ of a knot $\Gamma$ which is everywhere tangent to the field of hyperplanes $\operatorname{ker}(d z-y d x)$ and which represents the knot class $K$

$$
S\left(\Gamma_{\mathbb{C}}\right)+\left|W\left(\Gamma_{\mathbb{C}}\right)\right| \leq 2 g(K)-1 .
$$

It is a curious fact that the roles of $S$ and $W$ in (1.2) and (1.3) are reversed.

\section{C New invariants of framed holonomic knots}

Trace [4] showed that two knot diagrams $D$ and $D^{\prime}$ are regularly isotopic if and only if they represent the same knot class, $W(D)=W\left(D^{\prime}\right)$, and $S(D)=S\left(D^{\prime}\right)$. The classification problem for framed holonomic knots resembles the problem of classifying knot diagrams up to regular isotopy in the following way. Regular isotopy is knot diagram isotopy without first Reidemeister moves (the move which projects to a cusp-instance) and framed holonomic isotopy is holonomic isotopy without the holonomic first Reidemeister move, see Figure 2.

Theorem 1.3 On the space of framed holonomic knots with Whitney index equal to $m$, the invariant $S$ splits. More precisely, to each framed holonomic knot $C$ with $W(C)=m$ there is associated $n$, where $n$ is the largest integer with $n \leq \frac{|m|}{2}$, integers $S_{1}(C), \ldots, S_{n}(C)$, which are invariant under framed holonomic isotopy. Moreover,

$$
S(C)=\sum_{j=1}^{n} S_{j}(C)
$$

The invariants $S_{j}$ are defined in Definition 4.1 and Theorem 1.3 is proved in Subsection 4.A.

In Section 5 we give examples of framed holonomic knots representing the same knot class, with the same $W$ and $S$ but which are distinguished up to framed holonomic isotopy by the invariants $S_{j}$. This shows that the classification of framed holonomic knots up to framed holonomic isotopy is more refined than the regular isotopy classification of their diagrams. This result should be compared to the result of Birman and Wrinkle mentioned in Subsection 1.A.

\section{D Holonomic regular homotopy}

A holonomic regular homotopy is a continuous 1-parameter family of regular holonomic plane curves. 
Proposition 1.4 Two regular holonomic plane curves are holonomically regularly homotopic if and only if they have the same Whitney index.

Proposition 1.4 is proved in Subsection 2.B. If the holonomic requirements in Proposition 1.4 are removed one obtains the classical Whitney-Graustein theorem [6]. The proof we present is independent of this theorem.

\section{Diagrams of holonomic knots and Reidemeister moves}

For the readers convenience, basic facts on the geometry of diagrams of holonomic knots are presented. For proofs of these facts, see [5], Proposition 1.

Let $f: S^{1} \rightarrow \mathbb{R}$ be a generic function. Then the $x_{0} x_{1}$-diagram $c$ of the framed holonomic knot $C$ associated to $f$ has the following properties:

P1 $c$ is a regular curve and if $p$ is a point on $c$ in the upper (lower) half plane and $v$ is the unit tangent of $c$ at $p$ then $\left\langle v, \partial_{x_{0}}\right\rangle>0\left(\left\langle v, \partial_{x_{0}}\right\rangle<0\right)$, where $\langle$,$\rangle denotes the standard inner product on \mathbb{R}^{2}$.

P2 $c$ meets the $x_{0}$-axis at right angles at a finite number of points corresponding to the local extrema of $f$. The curvature of $c$ at such a point $p$ does not vanish and if $p$ corresponds to maximum (minimum) of $f$ the unit tangent of $c$ at $p$ equals $-\partial_{x_{1}}\left(\partial_{x_{1}}\right)$.

P3 The only self intersection points of $c$ are transverse double points which lie in the region $\left\{\left(x_{0}, x_{1}\right): x_{1} \neq 0\right\}$. The crossing number of a double point in the upper (lower) half plane is negative (positive) with respect to the orientation $d x_{0} \wedge d x_{1} \wedge d x_{2}>0$.

In generic 1-parameter families of framed holonomic knots the diagram changes by planar isotopy which preserve properties P1-3 above except for a finite number of instances where one of the bifurcations in Figure 1 occur. Note that the $\Omega_{2}$-moves always occur on the $x_{0}$-axis. The signs on the $\Omega_{2}$-moves refer to the signs of the product of the second derivatives at the extrema meeting at the self-tangency moment of the function defining the holonomic knot. The $\Omega_{3}$-move depicted occurs either in the upper- or lower half plane.

If the word framed above is omitted the corresponding result is: In generic 1parameter families of holonomic knots the diagram changes by planar isotopy which preserve properties $\mathbf{P 1 -} \mathbf{3}$ above except for a finite number of instances where one of the bifurcations in Figures 1 or 2 occur. The signs of the $\Omega_{1}$-moves 

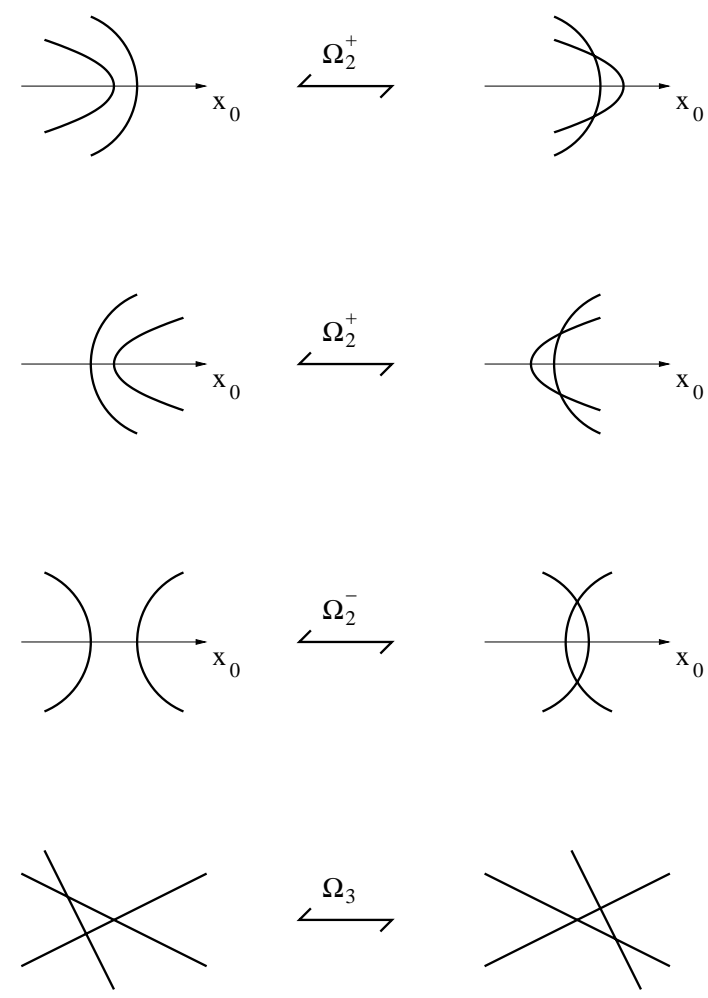

Figure 1: Framed holonomic Reidemeister moves
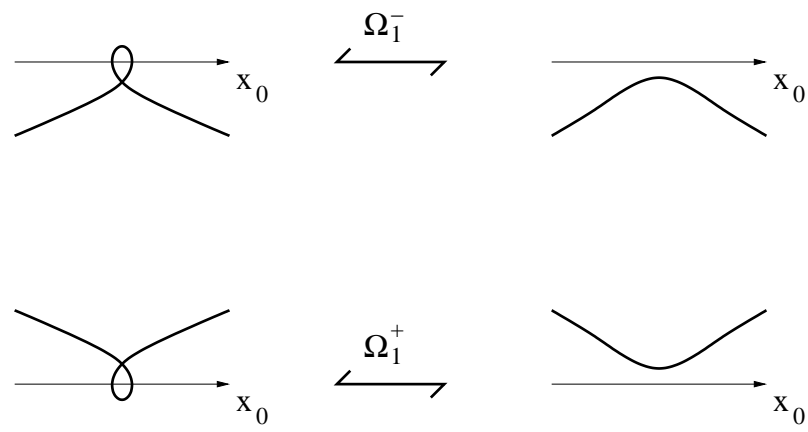

Figure 2: Holonomic versions of the first Reidemeister move

in Figure 2 refer to the sign of $x_{1}$ in the half plane where a double point is born or vanishes.

If we further omit the condition that the holonomic curve be an embedding then

Algebraic 83 Geometric Topology, Volume 2 (2002) 
the list of diagram-bifurcations would be further extended and include also the move in Figure 3 (which might change the knot class of the holonomic curve). The signs on the $\Omega_{0}$-moves in Figure 3 refer to the half plane where double

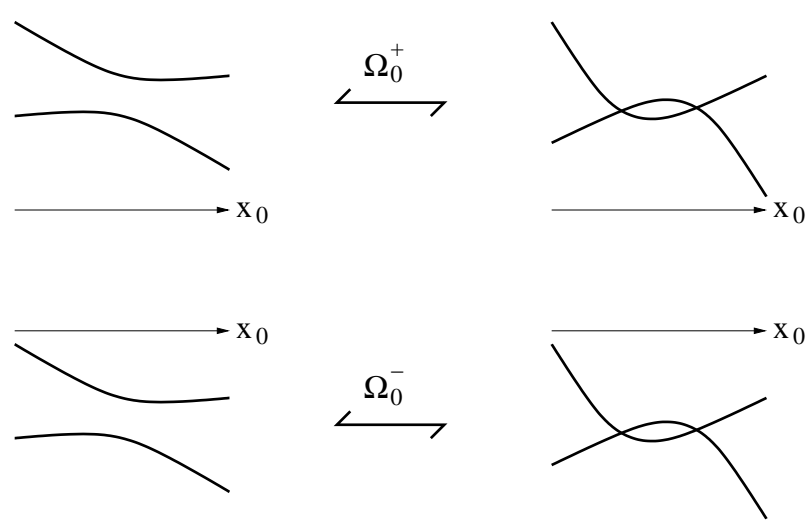

Figure 3: Framed holonomic crossing move

points are born or vanish.

\section{A Proof of Proposition 1.1}

Let $\pi\left(x_{0}, x_{1}, x_{2}\right)=\left(x_{0}, x_{1}\right)$. Let $f$ be a generic function on the circle, let $C$ be its associated holonomic knot, and let $c=\pi(C)$. To see that $W(C)<0$ compute the Whitney index by looking at points $p$ on $c$ where the unit tangent equals $\partial_{x_{1}}$. These correspond to minima of $f$ and all of them contribute -1 to $W$. The second statement is immediate.

To create a holonomic knot $C$ with $W(C)=m, m \leq-2$ and $S(C)=n$, start from the holonomic unknot diagram (which is just the unit circle). If $n \geq 0$ $(n<0)$ apply $\Omega_{1}^{+}\left(\Omega_{1}^{-}\right) m-1$ times in such a way that the resulting diagram contains $m-1$ consecutive loops along the $x_{0}$-axis. The resulting holonomic knot has $W=m$ and $S=-(m-1)$ if $n \geq 0(S=m-1$ if $n<0)$. Finally, if $n \geq 0$ apply $\Omega_{0}^{-} \frac{n+m-1}{2}$ times and if $n<0$ apply $\Omega_{0}^{+} \frac{n+m-1}{2}$ times to create new double points. The resulting holonomic knot $C$ then has $W(C)=m$ and $S(C)=n$, as desired.

\section{B Proof of Proposition 1.4}

Let $f$ be a function with associated holonomic plane curve $c_{f}$ which is an immersion. If $\phi$ is a diffeomorphism of $S^{1}$ then also $f \circ \phi$ gives rise to a regular 
holonomic plane curve.

Let $g$ be a function with regular plane holonomic curve $c_{g}$ with $W\left(c_{f}\right)=W\left(c_{g}\right)$. Perturb $f$ and $g$ so that they become Morse functions. Then the proof of Proposition 1.1 implies that they have the same number of local extrema. Let $\phi_{s}, 0 \leq s \leq 1$ be a diffeotopy of $S^{1}$ which moves each critical point of $f$ to a critical point of $g$ of the same index. Then the critical sets of $\hat{g}=g \circ \phi_{1}$ and of $f$ agree. Moreover, if $t$ is local maximum (minimum) of $f$ then it is a local maximum (minimum) of $g \circ \phi_{1}$. Let $(s, r)$ be coordinates on the cylinder $S^{1} \times \mathbb{R}$ and consider the vector field $V(s, r)=(f(s)-\hat{g}(s)) \partial_{r}$. Let $\Phi^{\rho}$ be the flow of $V$. If $\hat{g}_{\rho}$ is the function with graph $\Phi^{\rho}\left(\Gamma_{\hat{g}}\right)$, where $\Gamma_{\hat{g}}$ is the graph of $\hat{g}$, then $\hat{g}_{\rho}$ has a regular associated holonomic curve for each $\rho \leq 1$ and $\hat{g}_{1}=f$.

These two deformations together give the desired holonomic regular homotopy.

\section{Holonomic knots and front projections of Legen- drian knots}

\section{A The front and complex projections of a Legendrian knot}

Let $\Gamma$ be a knot in $\mathbb{R}^{3}$ with coordinates $(x, y, z)$ everywhere tangent to the plane field $\{\operatorname{ker}(d z-y d x)\}$. That is, $\Gamma$ is a Legendrian knot. Assume moreover that $\Gamma$ is generic among Legendrian knots, then the projection $\Gamma_{F}$ of $\Gamma$ to the $x z$-plane is a curve with transverse double points, isolated cusps, and without vertical tangencies. Moreover, given any curve in the $x z$-plane with these properties, there exists a unique Legendrian knot which projects to this curve. We associate the following numbers to $\Gamma_{F}$ :

First we count cusps, let $\operatorname{Dcu}\left(\Gamma_{F}\right), \operatorname{Ucu}\left(\Gamma_{F}\right)$, and $\operatorname{Lcu}\left(\Gamma_{F}\right)$ denote the number of down-cusps, up-cusps, and left-cusps respectively of $\Gamma_{F}$, see Figure 4.

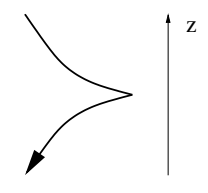

(A)

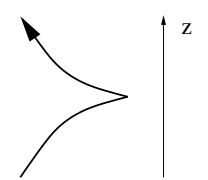

(B)

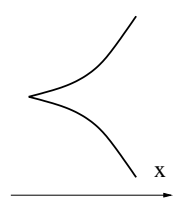

(C)

Figure 4: (A) Down-cusp, (B) Up-cusp, and (C) left-cusp. 
Second we count crossings, let $\operatorname{Ecr}\left(\Gamma_{F}\right)$ denote the number of crossing points where the tangent vectors has $x$-components of the same sign and $\operatorname{Ocr}\left(\Gamma_{F}\right)$ the number of crossing points where the tangent vectors has $x$-components of the opposite sign. See Figures 5 and 6 .
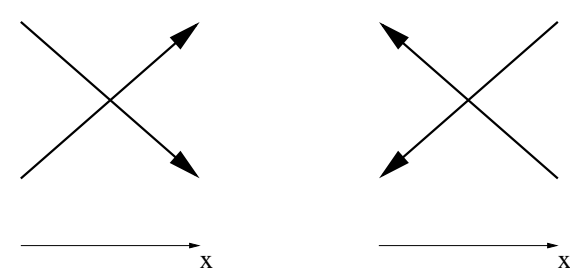

Figure 5: Crossing points with tangents with $x$-components of equal sign
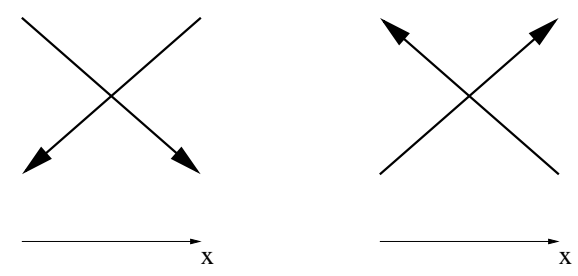

Figure 6: Crossing points with tangents with $x$-components of opposite signs

The projection $\Gamma_{\mathbb{C}}$ of $\Gamma$ to the $x y$-plane is a generic knot diagram. It is straightforward to check that the invariants $W\left(\Gamma_{\mathbb{C}}\right)$, where we use the orientation given by $d x \wedge d y$ in the $x y$-plane, and $S\left(\Gamma_{\mathbb{C}}\right)$, where we use the orientation given by $d x \wedge d y \wedge d z$ in space, can be computed from data of $\Gamma_{F}$ as follows,

$$
\begin{aligned}
W\left(\Gamma_{\mathbb{C}}\right) & =\frac{1}{2}\left(\operatorname{Dcu}\left(\Gamma_{F}\right)-\operatorname{Ucu}\left(\Gamma_{F}\right)\right), \\
S\left(\Gamma_{\mathbb{C}}\right) & =\operatorname{Ecr}\left(\Gamma_{F}\right)-\operatorname{Ocr}\left(\Gamma_{F}\right)-\operatorname{Lcu}\left(\Gamma_{F}\right) .
\end{aligned}
$$

\section{B Legendrian knots associated to a holonomic one}

Let $C$ be a framed holonomic knot. We associate two Legendrian knots $\Gamma^{+}$ and $\Gamma^{-}$, everywhere tangent to $\operatorname{ker}\left(d x_{1}-x_{2} d x_{0}\right)$, to $C$ by describing their front projections (in the $x_{0} x_{1}$-plane). The resulting Legendrian knots lie in $\mathbb{R}^{3}$ oriented by $d x_{0} \wedge d x_{2} \wedge d x_{1}>0$.

The first step in the construction of the fronts of $\Gamma^{+}$and $\Gamma^{-}$is the same in both cases: 
The points where the diagram of $C$ has vertical tangents are all confined to the $x_{0}$-axis. Replace neighborhoods of such points in the diagram with cusped arcs as described in Figure 7. The second step however differs:

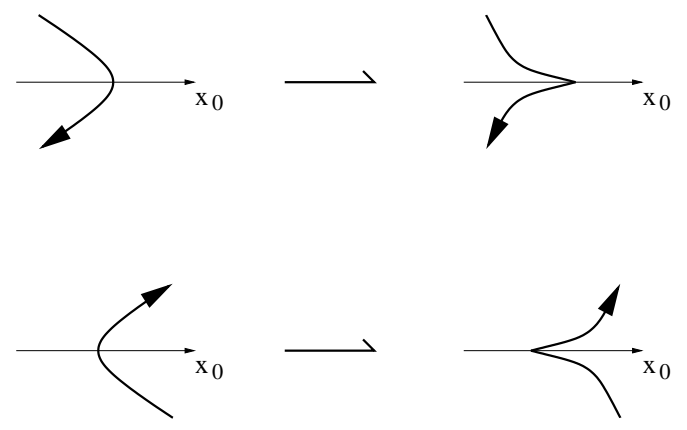

Figure 7: Replacing vertical tangencies with cusps

To obtain the front of $\Gamma^{+}$we insert a zig-zag as in Figure 8 at all crossings in the lower half plane and keep the crossings in the upper half plane as they are.

To obtain the front of $\Gamma^{-}$we insert a zig-zag as in Figure 9 at all crossings in the upper half plane and keep the crossings in the lower half plane as they are.

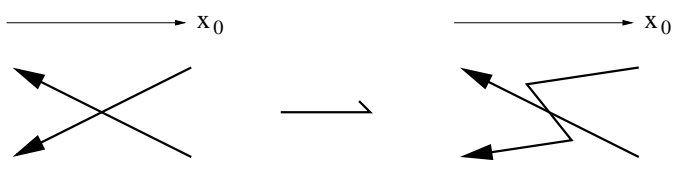

Figure 8: Inserting a zig-zag in the lower half plane

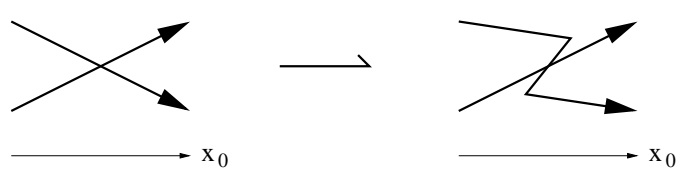

Figure 9: Inserting a zig-zag in the upper half plane

It is easy to check that $\Gamma^{-}\left(\Gamma^{+}\right)$is topologically isotopic to the knot $C$ in $\mathbb{R}^{3}$ equipped with the orientation $d x_{0} \wedge d x_{1} \wedge d x_{2}>0\left(d x_{0} \wedge d x_{1} \wedge d x_{2}<0\right)$.

Algebraic 63 Geometric Topology, Volume 2 (2002) 


\section{C Proof of Theorem 1.2}

Using $\Omega_{2}$-moves, we may obtain a closed braid representation of $K$. Equation (1.1) follows.

To prove $(1.2)$, let $H_{+}(C)\left(H_{-}(C)\right)$ denote the number of intersection points of $C$ in the upper (lower) half plane. Then $S(C)=H_{-}(C)-H_{+}(C)$. As noted before, $W(C)$ is the negative of the number of local minima of the function $f$ giving rise to $C$.

Let $\Gamma^{+}$and $\Gamma^{-}$be the Legendrian knots associated to $C$ as in Subsection 3.B. Then

$$
\begin{aligned}
& \operatorname{Dcu}\left(\Gamma_{F}^{+}\right)=-W(C)+2 H_{-}(C), \\
& \operatorname{Dcu}\left(\Gamma_{F}^{-}\right)=-W(C)+2 H_{+}(C), \\
& \operatorname{Ucu}\left(\Gamma_{F}^{+}\right)=\operatorname{Ucu}\left(\Gamma_{F}^{-}\right)=-W(C),
\end{aligned}
$$

and hence

$$
\begin{aligned}
& W\left(\Gamma_{\mathbb{C}}^{+}\right)=H_{-}(C), \\
& W\left(\Gamma_{\mathbb{C}}^{-}\right)=H_{+}(C) .
\end{aligned}
$$

Also,

$$
\begin{aligned}
& \operatorname{Lcu}\left(\Gamma_{F}^{+}\right)=-W(C)+H_{-}(C), \\
& \operatorname{Lcu}\left(\Gamma_{F}^{-}\right)=-W(C)+H_{+}(C), \\
& \operatorname{Ecr}\left(\Gamma_{F}^{+}\right)=\operatorname{Ocr}\left(\Gamma_{F}^{-}\right)=H_{+}(C), \\
& \operatorname{Ecr}\left(\Gamma_{F}^{-}\right)=\operatorname{Ocr}\left(\Gamma_{F}^{+}\right)=H_{-}(C),
\end{aligned}
$$

and hence

$$
\begin{aligned}
& S\left(\Gamma_{\mathbb{C}}^{+}\right)=H_{+}(C)-2 H_{-}(C)+W(C), \\
& S\left(\Gamma_{\mathbb{C}}^{-}\right)=H_{-}(C)-2 H_{+}(C)+W(C) .
\end{aligned}
$$

Combining (3.3) and (3.5), respectively (3.4) and (3.6) with the Bennequin inequality (1.3) yields

$$
\begin{gathered}
-S(C)+W(C) \leq 2 g(K)-1 \text { and } \\
S(C)+W(C) \leq 2 g(K)-1,
\end{gathered}
$$

since the genus does not depend on the orientation of the ambient space. The theorem follows.

Algebraic $6 \mathcal{G}$ Geometric $\mathcal{T}$ opology, Volume 2 (2002) 


\section{Splitting the self linking number}

Consider the diagram of a framed holonomic knot $C$. The $x_{0}$-axis divides the diagram into cyclically ordered $\operatorname{arcs}\left(X_{i}, Y_{i}\right), i=1, \ldots, m$, where the $X_{i}$ lies in the upper half plane, the $Y_{i}$ in the lower, and where $-m=W(C)$.

Let $\left(A_{i}, A_{j}\right)=\left(X_{i}, X_{j}\right)$ or $\left(A_{i}, A_{j}\right)=\left(Y_{i}, Y_{j}\right)$ where $i \neq j$. Define

$\delta\left(A_{i}, A_{j}\right)= \begin{cases}1 & \text { if } \partial A_{i} \text { is contained in an unbounded component of } \mathbb{R}-\partial A_{j}, \\ 0 & \text { otherwise. }\end{cases}$

Define

$$
N\left(A_{i}, A_{j}\right)=\left|A_{i} \cap A_{j}\right|+\delta\left(A_{i}, A_{j}\right),
$$

where $|S|$ denotes the number of points in the set $S$.

Let $\tilde{X}_{i}$ and $\tilde{Y}_{i}$ denote the preimages of $X_{i}$ and $Y_{i}$, for $i=1, \ldots, m$. Let $x_{i}$ and $y_{i}$ denote the midpoints of $\tilde{X}_{i}$ and $\tilde{Y}_{i}$, respectively.

Consider two $\operatorname{arcs} X_{i}$ and $X_{j}, i \neq j$. Let $\gamma\left(x_{i}, x_{j}\right)$ denote the unique oriented arc connecting $x_{i}$ to $x_{j}$ with orientation agreeing with that of the circle. Define the cyclic distance of $X_{i}$ and $X_{j}$ as

$$
d\left(X_{i}, X_{j}\right)=\min \left\{\left|\gamma\left(x_{i}, x_{j}\right) \cap\left\{y_{1}, \ldots, y_{m}\right\}\right|,\left|\gamma\left(x_{j}, x_{i}\right) \cap\left\{y_{1}, \ldots, y_{m}\right\}\right|\right\} .
$$

Define the cyclic distance of $\operatorname{arcs} Y_{i}$ and $Y_{j}$ analogously.

Definition 4.1 Define

$$
S_{k}(C)=\frac{1}{2}\left(\sum_{\left\{\left(Y_{i}, Y_{j}\right): d\left(Y_{i}, Y_{j}\right)=k\right\}} N\left(Y_{i}, Y_{j}\right)-\sum_{\left\{\left(X_{i}, X_{j}\right): d\left(X_{i}, X_{j}\right)=k\right\}} N\left(X_{i}, X_{j}\right)\right) .
$$

Remark 4.2 In terms of defining functions, the terms in the definition of $S_{k}$ can be interpreted as follows. Let $f: S^{1} \rightarrow \mathbb{R}$ be a function with associated framed holonomic knot $C$. Consider $f$ as a periodic function with period $T$ such that $f(0)=f(T)$ is the global minimum of $f$. Let $\Gamma_{f} \subset[0, T] \times \mathbb{R} \subset$ $\mathbb{R}^{2}$ denote the graph of $f$. Then the arcs $X_{i}\left(Y_{i}\right)$ are the holonomic curves corresponding to restrictions of $f$ to subintervals of $[0, T]$, where $f$ is increasing (decreasing). If $(x, y)$ are coordinates on $\mathbb{R}^{2}$ then $\left|A_{i} \cap A_{j}\right|$ equals the number of lines $l_{a}=\{y=a\}, a \in \mathbb{R}$ which intersect $A_{i}$ and $A_{j}$ at equal angles, and $\delta\left(A_{i}, A_{j}\right)=1$ if no $l_{a}$ intersect both $A_{i}$ and $A_{j}$, otherwise it is 0 . 


\section{A Proof of Theorem 1.3}

We check that $S_{k}$ is invariant under framed holonomic Reidemeister moves. For $\Omega_{3}$ this is immediate.

An $\Omega_{2}^{+}$-move involving two $\operatorname{arcs} X$ and $X^{\prime}$ with distance $d\left(X, X^{\prime}\right)=k$ involves also two $\operatorname{arcs} Y$ and $Y^{\prime}$ with $d\left(Y, Y^{\prime}\right)=k$. At the move $\delta\left(X, X^{\prime}\right)$ and $\delta\left(Y, Y^{\prime}\right)$ are unchanged and the change in $\left|X \cap X^{\prime}\right|$ and $\left|Y \cap Y^{\prime}\right|$ are the same. Hence $S_{k}$ remains constant.

At an $\Omega_{2}^{-}$-move involving $\operatorname{arcs} X$ and $X^{\prime}, Y$ and $Y^{\prime}$ the change in $\left|X \cap X^{\prime}\right|$ and $\left|Y \cap Y^{\prime}\right|$ equals the change in $\delta\left(X, X^{\prime}\right)$ and $\delta\left(Y, Y^{\prime}\right)$, respectively. Hence $S_{k}$ remains constant.

To prove (1.4) note that by using the $\Omega_{2}$-moves we may move any framed holonomic knot diagram in such a way that its diagram is a closed braid with braid-axis parallel to the $x_{2}$-direction. (The linking number of this axis oriented in the positive $x_{2}$-direction and the holonomic knot with its natural orientation is negative.) Under such deformations both $S$ and $S_{1}, \ldots, S_{n}$, remain constant. Moreover for a diagram which is a closed braid $\delta\left(X_{i}, X_{j}\right)=\delta\left(Y_{i}, Y_{j}\right)=0$ for all $i, j$. Hence both the left and right hand sides of (1.4) are equal to the difference of the number of double points in the lower and upper half planes.

\section{$5 \quad$ Examples}

The framed holonomic knots $K_{1}$ in Figure 10 and $K_{2}$ in Figure 11 both represent the unknot. Since $S\left(K_{1}\right)=S\left(K_{2}\right)=-1$ and $W\left(K_{1}\right)=W\left(K_{2}\right)=-4$, $K_{1}$ and $K_{2}$ are regularly isotopic. Since $K_{1}$ is a closed braid $\delta\left(X_{i}, X_{j}\right)=0=$ $\delta\left(Y_{i}, Y_{j}\right)$ for all $i, j$. Noting that all three intersection points in the diagram of $K_{1}$ in the upper half plane are intersections between arcs of cyclic distance 1 , and that the two intersection points in the lower half plane are intersections of arcs of cyclic distance 1 respectively 2 , we conclude that $S_{1}\left(K_{1}\right)=-2$ and $S_{2}\left(K_{1}\right)=1$. A similar calculation gives $S_{1}\left(K_{2}\right)=0$ and $S_{2}\left(K_{2}\right)=-1$. Hence $K_{1}$ and $K_{2}$ are not framed holonomically isotopic.

The framed holonomic knots $K_{3}$ in Figure 12 and $K_{4}$ in Figure 13 both represent the connected sum of the trefoil and its mirror image. Since $S\left(K_{3}\right)=$ $S\left(K_{4}\right)=-1$ and $W\left(K_{3}\right)=W\left(K_{4}\right)=-4, K_{3}$ and $K_{4}$ are regularly isotopic. However, $S_{1}\left(K_{3}\right)=0$ and $S_{2}\left(K_{3}\right)=-1$ but $S_{1}\left(K_{4}\right)=-4$ and $S_{2}\left(K_{4}\right)=3$ so $K_{3}$ and $K_{4}$ are not framed holonomically isotopic. 


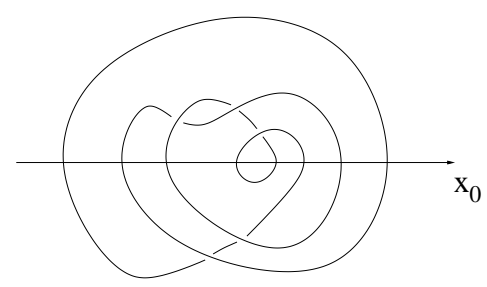

Figure 10: The framed holonomic knot $K_{1}$

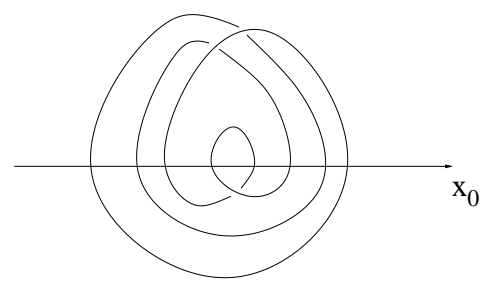

Figure 11: The framed holonomic knot $K_{2}$

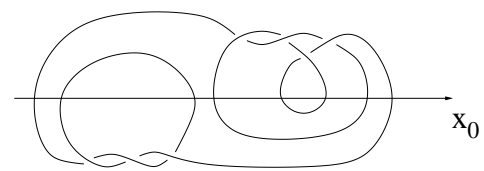

Figure 12: The framed holonomic knot $K_{3}$

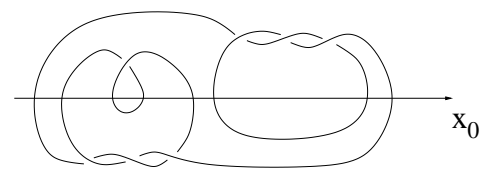

Figure 13: The framed holonomic knot $K_{4}$

\section{References}

[1] D. Bennequin, Entrelacements et équations de Pfaff, Soc. Math. de France, Astérisque 107-108 (1983) 87-161.

[2] J. S. Birman and N. C. Wrinkle, Holonomic and Legendrian parametrizations of knots, J. Knot Theory Ramifications 9 (2000), 293-309.

[3] L. Kauffman, Knots and Physics, World Scientific Publishing Co., Inc., River Edge, NJ (1991).

Algebraic 63 Geometric Topology, Volume 2 (2002) 
[4] B. Trace, On the Reidemeister moves of a classical knot, Proc. Amer. math. Soc. 89 (1983) 722-724.

[5] V. A. Vassiliev, Holonomic links and Smale principles for multisingularities, J. Knot Theory Ramifications 6 (1997) 115-123.

[6] H. Whitney, On regular closed curves in the plane, Composito Math. 4 (1936) $276-284$.

Department of Mathematics, Uppsala University

P.O. Box 480, 75106 Uppsala, Sweden

and

Département de Mathématiques et Informatique, Ecole Normale Supérieure de Lyon 46 allée d'Italie, 69364 Lyon Cédex 07, France

Email: tobias@math.uu.se, mwolff@ens-lyon.fr

Received: 11 December 2001 Revised: 17 May 2002 